\title{
Prevalence and risk factors of neonatal pneumonia in China: A longitudinal clinical study.
}

\author{
Lihong Yang $^{\text {**, Ying Zhang }}{ }^{2}$, Xuehui Yu ${ }^{3}$, Man Luo ${ }^{1}$ \\ ${ }^{1}$ Department of Nursing, Medical College of ChiFeng University, Chifeng, Inner Mongolia, PR China \\ ${ }^{2}$ School of Nursing, Jinzhou Medical University, Jinzhou, Liaoning, PR China \\ ${ }^{3}$ The Third Department of Orthopedics, the Central Hospital of Ningcheng, Chifeng, Inner Mongolia, PR China
}

\begin{abstract}
Aim: To evaluate the prevalence of neonatal pneumonia in China, and to identify the associated risk of developing neonatal pneumonia.

Subject and method: In this longitudinal clinical study, we identified a total of the 953 medical records of neonatal who were admitted at Medical college of ChiFeng University, China between March 2010 to 2017. Parents of each neonate patient were instructed to complete questionnaire about the symptoms associated with pneumonia. Potential risk factors were assessed using a hospital record of all childbirths occurred between March 2010 to 2017.

Results: Medical records of 953 neonate (male: 553, female: 400) with mean (SD) age of 2.4 (1.2) days who were admitted during March 2010 to 2017 were studied. Incidence of pneumonia among neonates was found to be $14 \%$. Our statistical analysis results showed that weight at the time of birth $(p=0.032)$, poor prenatal care $(\mathrm{p}=0.02), \mathrm{C}$-section surgery and delivery $(\mathrm{p}=0.02)$, home delivery of baby at home $(p<0.05)$, delivery of baby by unexperienced peoples $(p<0.05)$, new-born resurgence $(p<0.05)$, fever at birth $(p<0.05)$, gynaecological problem during pregnancy $(p<0.005)$, vulgar sensing fluid $(p<0.05)$, extended break of tissue $(p<0.005)$, sustained labour $(p=0.023)$ were meaningfully related to pneumonia. Conclusion: In China, a total of $14 \%$ of neonates had experienced pneumonia. Among identified risk factors, the multivariate regression analysis suggested that inadequate poor prenatal care, home delivery of baby at home, fever at birth, and gynaecological problem during pregnancy, sustained labor period were found as noteworthy risk factors of neonatal pneumonia among Chinese patients.
\end{abstract}

Keywords: Pneumonia, Neonates, Chinese patients, Pregnancy, Prevalence.

\section{Introduction}

Neonatal Pneumonia (NP) is most common cause of morbidity, mortality, and increase economic burden to their family [1,2]. It is a key reason of death in neonates: over 1.4 million deaths per year are attributable to pneumonia among the children [2-5]. Epidemiological studies have shown that the prevalence of NP in neonates is higher than assumed [5-7].

It has been seen that parents or care takers are unable to seek primary care for their children at the time of pneumonia development, which may be due to poor understanding of sign and symptoms of pneumonia including financial limits, lack of awareness and poor availability of health care centers $[7,8]$. There was many previously identified risk which plays an important role in development of NP, which includes ethnicity, family history, weight, smoking during pregnancy, age of women before and after pregnancy, gender, education level of parents, history of pneumonia in mother, number of brother and sisters and economic status [9-11].
Since causes of NP are remained uncertain although many efforts were made to discover the possible reasons, there was no recognized and active procedure to avoid the NP. Hence, understanding of possible risk factor is important which really helps in preventing NP incidence rate in future. Earlier, the incidence rate of NP in Chinese neonates was not assessed in any longitudinal study. Hence, we intended to conduct a longitudinal study which assesse the incidence rate and possible risk factor in developing NP in China. We precisely assessed the role of mother's conditions and history including several other factors which could play an important role in etiology of developing NP. The aim of this study was to assess the prevalence of NP in China neonates, and to identify the associated risk factors in developing NP.

\section{Subject and Method}

In this longitudinal clinical study, we identified a total of the 953 medical records of neonatal who were admitted at Medical 
College of ChiFeng University, China between March 2010 to 2017. Online database of Medical college of ChiFeng University was used to gather requisite data to achieve the objective of this study. All the neonates patients who were admitted in our hospital were undergo laboratory investigation including culture test to confirm the presence of pneumonia. Bacterial species includes gram positive, gram negative and other potential bacterial infection were ruled out. Parents of each neonate patient were instructed to complete questionnaire about the symptoms associated with pneumonia. Potential risk factors were assessed using a hospital record of all childbirths happening between March 2010 to 2017. This study and study protocol was review and approved by institutional ethics committee of Medical college of ChiFeng University, China, and written informed consent from parents of each neonates was taken before enrolling them into this study. All the ethical principles which are laid down in Helsinki Declaration of 1964, as revised in 2013 were followed during the conduct of this observational longitudinal study.

Parents, specially the mother of all neonates whose medical records were reviewed and asked to fill the questionnaire which capture all pregnancy related characteristics including demography and maternal characteristics. The data related to consequence of pregnancy was recorded from medical archives of each pregnant woman. We also assessed the collected data related to other potential factor which might played a critical role in development of NP. Since this was an observational study, no formal sample size calculation was executed. However, we have planned to include medical records of at least 500 Chinese neonates admitted in our hospital. Risk factors of neonatal pneumonia between neonatal pneumonia group and non-neonatal pneumonia group were analyzed by unpaired " $t$ " test except for gender, which was analyzed by Chi-square test. Potential risk factors for neonatal pneumonia were analyzed by logistic regression analysis. In all cases, a P value less than 0.05 were as difference between comparisons of interest was statistically significant. Statistical analysis was performed using version 6.2 of Graph Pad Prism.

\section{Results}

During the $7 \mathrm{y}$ time period of the study, 953 hospitalized neonates underwent laboratory investigation. Out of 953 hospitalized neonates, blood culture test of 131 neonates were positive for the presence of bacterial pneumonia. Majority of admitted patients were boys (male: 553, female: 400) with mean (SD) age of 2.4 (1.2) days. Our study result suggested that the prevalence of pneumonia among neonates was found to be approximately $14 \%$ (131/953 patients). Our statistical analysis results showed that weight at the time of birth $(p=0.032)$, poor prenatal care $(p=0.02), C$-section surgery and delivery $(p=0.02)$, home delivery of baby at home $(p<0.05)$, delivery of baby by unexperienced peoples $(p<0.05)$, new-born resurgence $(p<0.05)$, fever at birth $(p<0.05)$, gynaecological problem during pregnancy $(\mathrm{p}<0.005)$, vulgar sensing fluid $(p<0.05)$, extended break of tissue $(p<0.005)$, sustained labour $(p=0.023)$ were meaningfully related to pneumonia (Table 1). Considering maternal characteristic of neonates who had NP, we found that significantly greater number of women in NP group were obese than the mother whose baby don't have NP. This shows that the majority of mother of NP were overweight at the time of delivery. We also observed that the mother of NP group had pre-mature delivery when compared to mother of non-NP group. C-section delivery was also higher in NP group than non-NP group of women. In NP groups, number of proteinuria cases was higher in comparison to nonNP groups. This may be due to the family history of proteinuria in women with NP groups as compared with NP groups. Incidence of NP was higher among women who often do smoking than women who were non-smoker.

Table 1. Risk factors of neonatal pneumonia between neonatal pneumonia group and non-neonatal pneumonia group.

\begin{tabular}{|c|c|c|c|}
\hline Parameters & $\begin{array}{l}\text { Neonatal pneumonia group } \\
(\mathrm{N}=131)\end{array}$ & $\begin{array}{l}\text { Non-Neonatal pneumonia group } \\
(\mathrm{N}=\mathbf{8 2 2})\end{array}$ & $P$ value \\
\hline Age (days), Mean (SD) & $1.2(0.8)$ & $2.9(0.8)$ & $<0.05$ \\
\hline Gender (Boy/Girl), \% & $70 / 33$ & $82 / 18$ & $<0.05^{*}$ \\
\hline Birth weight, Mean (SD) & $3.3(1.1)$ & $5.6(2.1)$ & $<0.05$ \\
\hline \multicolumn{4}{|l|}{ Inadequate antenatal care } \\
\hline Yes $(\%)$ & 80 & 22 & $<0.05$ \\
\hline No (\%) & 20 & 78 & \\
\hline \multicolumn{4}{|c|}{ C-section surgery and delivery } \\
\hline Yes $(\%)$ & 68 & 39 & $<0.05$ \\
\hline No $(\%)$ & 32 & 61 & \\
\hline \multicolumn{4}{|l|}{ Home delivery } \\
\hline Yes (\%) & 68 & 39 & $<0.05$ \\
\hline
\end{tabular}




\begin{tabular}{|c|c|c|c|}
\hline No (\%) & 32 & 61 & \\
\hline \multicolumn{4}{|c|}{ Delivery by untrained personnel } \\
\hline Yes $(\%)$ & 40 & 12 & $<0.05$ \\
\hline No (\%) & 60 & 88 & \\
\hline \multicolumn{4}{|c|}{ Neonatal resuscitation } \\
\hline Yes $(\%)$ & 32 & 17 & $<0.05$ \\
\hline No $(\%)$ & 88 & 83 & \\
\hline \multicolumn{4}{|c|}{ Fever at birth } \\
\hline Yes (\%) & 40 & 11 & $<0.05$ \\
\hline No $(\%)$ & 60 & 89 & \\
\hline \multicolumn{4}{|c|}{ Gynecological problem during pregnancy } \\
\hline Yes (\%) & 25 & 12 & $<0.05$ \\
\hline No $(\%)$ & 75 & 88 & \\
\hline \multicolumn{4}{|c|}{ Foul smelling liquor } \\
\hline Yes $(\%)$ & 15 & 8 & $<0.05$ \\
\hline No (\%) & 85 & 92 & \\
\hline \multicolumn{4}{|c|}{ Prolonged rupture of membrane } \\
\hline Yes (\%) & 23 & 18 & $<0.05$ \\
\hline No (\%) & 77 & 82 & \\
\hline \multicolumn{4}{|c|}{ Prolonged labour } \\
\hline Yes (\%) & 32 & 21 & $<0.05$ \\
\hline No (\%) & 68 & 89 & \\
\hline
\end{tabular}

Values are expressed as mean (SD) for age and weight at the time of birth, rest all are expressed as percentage of patients in each category. $P$ value was calculated using Unpaired "t" test except for gender." $\mathrm{p}$ value was calculated using chi-square test. Abbreviation: SD: Standard Deviation.

Among identified risk factors, the multivariate regression analysis suggested that inadequate poor prenatal care, home delivery of baby at home, fever at birth, gynaecological problem during pregnancy (OR 26), and delay labor period were found as noteworthy risk factors of neonatal pneumonia among Chinese patients (Table 2).

Table 2. Potential risk factor for neonatal pneumonia using logistic regression analysis.

\begin{tabular}{lll}
\hline Parameters & Odd ratio 95\% Cl & P value \\
\hline Birth weight & $2.23(2.5-4.5)$ & $<0.05$ \\
\hline Inadequate antenatal care & $24.9(21.2-28.6)$ & $<0.0001$ \\
\hline C-section surgery and delivery & $3.13(3.3-4.6)$ & $<0.05$ \\
\hline Home delivery & $32.8(30.2-36.4)$ & $<0.0001$ \\
\hline Delivery by untrained personnel & $5.12(4.3-8.6)$ & $<0.05$ \\
\hline Neonatal resuscitation & $2.13(2.0-8.6)$ & $<0.05$ \\
\hline Fever at birth & $27.9(23.3-29.5)$ & $<0.0001$ \\
\hline
\end{tabular}

\begin{tabular}{lcc}
\hline Gynecological problem during pregnancy & $26.5(20.6-30.4)$ & $<0.0001$ \\
\hline Foul smelling liquor & $3.23(2.3-6.3)$ & $<0.05$ \\
\hline Prolonged rupture of membrane & $2.13(2.0-5.6)$ & $<0.05$ \\
\hline $\begin{array}{l}\text { Prolonged labour } \\
\text { P value was calculated using logistic regression analysis. Abbreviation: } \mathrm{Cl}\end{array}$ \\
\begin{tabular}{l} 
Confidence Interval. \\
\hline
\end{tabular}
\end{tabular}

\section{Discussion}

Neonatal pneumonia is a significant public health problem which is gaining increased awareness in most developed countries. It is a major cause of mortality among the children [1-7]. There was much previously identified risk which plays an important role in development of NP, which includes ethnicity, family history, weight, and smoking during pregnancy, age of women before and after pregnancy, gender, education level of parents, and history of pneumonia in mother [9-11]. Since causes of NP are remained uncertain although significant efforts was made to discover the possible reasons, hence, there was no recognized and active procedure to avoid 
the NP. Therefore the understanding of possible risk factor is important which really helps in preventing incidence of NP. Earlier, the incidence rate of NP in Chinese neonates was not assessed in any longitudinal study. Hence, we intended to conduct a longitudinal study which assess the incidence rate and possible risk factor in developing NP in China.

This is the first longitudinal study in China which collected data over the period of $7 \mathrm{y}$, and designed to evaluate the prevalence of neonatal pneumonia in China, and to identify the associated risk of developing neonatal pneumonia. All the neonates patients who were admitted in our hospital were undergone laboratory investigaton including culture test to confirm the presence of pneumonia. Potential risk factors were assessed using a hospital record of all childbirths occurred between March 2010 to 2017. Parents, specially the mother of all neonates whose medical records were reviewed and asked to fill the questionnaire which capture all pregnancy related characteristics including demography and maternal characteristics status. The data related to consequence of pregnancy was recorded from medical archives of each pregnant woman. Our study results showed that weight at the time of birth, poor prenatal care, C-section surgery and delivery, home delivery of baby at home, delivery of baby by unexperienced peoples, new-born resurgence, fever at birth, gynaecological problem during pregnancy, vulgar sensing fluid, extended break of tissue, sustained labour were meaningfully related to pneumonia. Among identified risk factors, the multivariate regression analysis suggested that inadequate poor prenatal care, home delivery of baby at home, fever at birth, gynaecological problem during pregnancy, sustained labour period were found as noteworthy risk factors of neonatal pneumonia among Chinese patients. Our study results encourage conducting a randomized, multinational, parallel group study to evaluate the risk factor of developing NP stratified by different characteristic of pregnancy. This will help to understand the relationship between maternal characteristics and occurrence of NP.

\section{Conclusion}

In China, a total of $14 \%$ of neonates had experienced pneumonia. Among identified risk factors, the multivariate regression analysis suggested that inadequate poor prenatal care, home delivery of baby at home, fever at birth, gynaecological problem during pregnancy, delay labor period were found as noteworthy risk factors of neonatal pneumonia among Chinese patients.

\section{Funding Support}

This study was supported by Inner Mongolia Autonomous Region Higher Education Science and Technology Research Project (No: NJZZ17295).

\section{References}

1. Barnett ED, Klein JO. Bacterial infections of the respiratory tract: Infectious diseases of the foetus and newborn infant. Clin Infect Dis 2001; 1006-1018.

2. Nissen MD. Congenital and neonatal pneumonia. Paediatrics Resp Rev 2007; 8: 195-203.

3. Duke T. Neonatal pneumonia in developing countries. Arch Dis Child Foetal Neonatal Ed 2005; 90: 2011-219.

4. Black RE, Cousens S, Johnson HL. Global, regional, and national causes of child mortality in 2008: A systematic analysis. Lancet 2010; 375: 1969-1987.

5. Liu L, Johnson HL, Cousens S, Perin J, Scott S, Lawn JE. Global, regional, and national causes of child mortality: an updated systematic analysis for 2010 with time trends since 2000. Lancet 2012; 379: 2151-2161.

6. Viscardi RM. Prenatal and postnatal microbial colonisation and respiratory outcome in preterm infants. Newborn Lung 2012; 6: 135-162.

7. Dear PRF, FIFE A. Pneumonia. Arnold, London 2003; 21 : 278-310.

8. Walker CL, Rudan I, Liu L, Nair H, Theodoratou E, Bhutta ZA. Global burden of childhood pneumonia and diarrhoea. Lancet 2013; 381: 1405-1416.

9. Barsam FJ, Borges GS, Severino AB, de Mello LM, da Silva AS, Nunes AA. Factors associated with communityacquired pneumonia in hospitalised children and adolescents aged 6 months to 13 years old. Eur J Pediatr 2013; 172: 493-499.

10. Thorn LK, Minamisava R, Nouer SS, Ribeiro LH, Andrade AL. Pneumonia and poverty: a prospective populationbased study among children in Brazil. BMC Infect Dis 2011; 11: 180 .

11. Scott JA, Brooks WA, Peiris JS, Holtzman D, Mulholland EK. Pneumonia research to reduce childhood mortality in the developing world. J Clin Invest 2008; 118: 1291-1300.

\section{*Correspondence to}

Lihong Yang

Department of Nursing

Medical College of ChiFeng University

PR China 